\title{
Kernos
}

Revue internationale et pluridisciplinaire de religion grecque antique

$10 \mid 1997$

Varia

\section{Pour une apologie de la transgression ? Esquisse d'une typologie}

\section{Alain Moreau}

\section{OpenEdition}

\section{Journals}

Édition électronique

URL : http://journals.openedition.org/kernos/650

DOI : $10.4000 /$ kernos. 650

ISSN : 2034-7871

\section{Éditeur}

Centre international d'étude de la religion grecque antique

\section{Édition imprimée}

Date de publication : 1 janvier 1997

Pagination : 97-110

ISSN : 0776-3824

\section{Référence électronique}

Alain Moreau, « Pour une apologie de la transgression? Esquisse d'une typologie », Kernos [En ligne], 10 | 1997, mis en ligne le 12 avril 2011, consulté le 04 mai 2019. URL : http://journals.openedition.org/ kernos/650; DOl : 10.4000/kernos.650 


\title{
Pour une apologie de la transgression?
}

\author{
Esquisse d'une typologie
}

Il est difficile de définir le mot grec bubris et de cerner avec précision son champ sémantique ${ }^{1}$. Aucun mot français ne lui répond exactement, aucun terme grec ne recouvre absolument cette notion. Les correspondants français les plus proches sont «transgression» et « démesure». Les dictionnaires rapprochent «transgression » de «désobéissance » ou «violation». Transgresser, c'est « passer outre ». On transgresse un interdit, un rite, les préceptes divins. Si l'on étudie le champ sémantique de parabainein, on trouve «transgresser, violer des lois, des serments, offenser une divinité par un acte de désobéissance ». Pâris et les Troyens qui ont enlevé Hélène sont comme les ravisseurs des petits de l'aigle. Ici parabantes (Esch., Ag., 59, sous la forme parabâsin) pourrait se rendre par «les coupables », mais il serait possible aussi de traduire le mot par «transgresseurs ». Le verbe de même racine buperbainein est très proche : " passer pardessus, franchir ». On transgresse, viole des serments, des lois, la justice. La «démesure » souligne une autre dimension du mot bubris, l'outrance, l'excès. Transgression et excès conduisent à la notion de « désordre », l'akosmia.

Pour affiner, il faudrait introduire aussi le concept de «faute » avec le verbe hamartanein qui, dans sa signification propre, signifie «manquer le but» au sens concret du terme (par exemple la flèche qui n'atteint pas sa cible). Au sens figuré le mot hésite entre l'erreur » et la «faute », le «manquement ». Lorsque le «manquement» concerne le domaine religieux, on rentre tout à fait dans le domaine de l'bubris. Il en est de même avec des synonymes d'bamartanein comme amplakiskein ou alitên : du sens de "commettre une faute », "s'égarer », on passe aisément à celui de «se rendre coupable » envers les hommes ou les dieux (violer un serment ou les ordres de Zeus). Il faudrait ajouter la

1 Éléments bibliographiques : Jan M. BREMER, Hamartia, Tragic Error in the Poetics of Aristotle and in Greek Tragedy, Amsterdam, 1969; Maurice DIRAT, L'Hybris dans la tragédie grecque, Thèse Toulouse 1972, repr. Univ, de Lille III, 1973; N.R.E. FISCHER, The Concept of Hybris in Greece from Homer to the Fourth Century, Oxford Univ. Pr., 1976, 2 vol,; ID., Hybris and Dishonor I, in GER, 23 (1976), p. 176-193; ID., Hybris. A Study in the Values of Honour and Shame in Ancient Greece, Warminster, Aris \& Philipps, 1992; Josua Johann FRAENKEL, Hybris, Thèse Utrecht, 1941; C. DEL GRANDE, Hybris. Colpa e castigo nell'espressione poetica e litteraria degli scrittori della Grecia antica. Da Omero a Cleante, Napoli, Riccardo Riccardi, 1947; J.T. HOOKER, The Original Meaning of $\hat{j} \beta p$ ţ, in $A B G, 19$ (1975), p. 125-137. 
notion d'injustice, de tort avec adikê̂n, « commettre une injustice, causer du tort », qui bascule aisément du domaine juridique au domaine moral et religieux, encore que le terme soit sans doute le plus profane de tous les termes cités et concerne le plus souvent les affaires de la polis. Mais il y a une Dikè divine, la Justice de Zeus. On ne doit pas oublier non plus la notion de jactance, emphase, vantardise avec kompeîn, ni, bien entendu, celle de l'impiété spécifique, du sacrilège avec asebeîn. Enfin il faut se souvenir que si le lecteur de la poésie grecque aurait tendance à comprendre bubris dans sa dimension religieuse, le lecteur de prose grecque, quant à lui, y verrait plutôt une notion profane. Il est clair que lorsqu'Euphilétos parle de l'bubris d'Ératosthène se glissant subrepticement dans le lit de sa jeune épouse, il donne au mot une signification légèrement différente de celle que donnent les poètes épiques et tragiques lorsqu'ils parlent de l'bubris de Typhon ou d'Ajax, fils d'Öllée.

C'est avec une pleine conscience de ces difficultés que je vais tenter néanmoins de dresser une esquisse de la typologie de l'bubris en insistant sur le concept de transgression.

\section{La transgression fondatrice. Transgresser pour créer}

Je dirais volontiers, parodiant Hésiode : «Au commencement fut la transgression ». C'est bien ce que nous dit l'Enûma Elish, l'épopée babylonienne de la Création ${ }^{2}$. Au commencement furent les eaux primordiales, Apsû, eau-douce, élément masculin, et Tiamat, la Mer, élément féminin. À la seconde génération il y eut Lahmu et Lahamu. À la troisième Anshar et Kishar. À la quatrième Anu. À la cinquième Éa-Nudimmud et d'autres enfants, non nommés. Les dieux de la cinquième génération, qui faisaient du « remue-ménage » dans l'Habitacle divin, troublent Tiamat. Une guerre se déclenche. Éa endort Apsû, le dépouille des talismans du pouvoir et le met à mort. Apsû devient le nom du palais du nouveau dieu souverain. Dans son palais, avec son épouse Damkina, Éa procrée Marduk, «le plus intelligent, le Sage des dieux, le Seigneur ». Mais une nouvelle guerre commence, car Marduk fabrique la Poussière, la fait emporter par la Tempête, provoque la Houle et trouble ainsi la déesse primordiale Tiamat et les dieux qui sont ses rejetons. Parmi ceux-ci Tiamat choisit Qingu (ou Kingu) comme époux et le met à la tête de ses armées, dans lesquelles on trouve une impressionnante série de monstres mis au monde par le ventre fécond et maléfique de Tiamat. Devant une telle puissance les autres dieux s'avèrent incapables de tenir. Ils font appel à Marduk qui négocie son aide contre le pouvoir souverain. Au terme d'un combat épique entre Tiamat et Marduk, le jeune dieu triomphe, met à mort la déesse primordiale. C'est à partir de son cadavre qu'il crée l'univers, "gigantesque sphéroïde creux ». Quant à l'humanité,

2 Jean BOTTÉRO et Samuel Noah KRAMER, Lorsque les dieux faisaient l'bomme. Mytbologie mésopotamienne, Paris, Gallimard, 1989, p. 602-679. 
il lui donne naissance à partir du sang de Qingu. Puis il fait procéder à l'érection de Babylone. On procède à l'investiture solennelle de Marduk. Le poème s'achève par la glorification du nouveau souverain du Monde.

C'est un schéma semblable qu'offre la Théogonie d'Hésiode. Au commencement fut Chaos. Puis Gaia, Éros, Érèbe, Nuit. À la troisième génération, de Nuit naissent Éther et Lumière du Jour; de Gaia naît Ouranos. De l'union de Gaia et d'Ouranos naissent, entre autres, les Titans, dont Cronos. Cronos châtre son père Ouranos et prend sa place. Uni à Rhéia, il engendre de nouveaux dieux, dont le dernier est « le grand Zeus », « son fils invincible et impassible ». Celui-ci triomphe de Cronos et s'empare du trône. Zeus reste en place à jamais malgré la révolte des Titans et de Typhon (sans compter la Gigantomachie, dont Hésiode ne parle pas, mais qui est certainement ancienne).

Dans la théogonie babylonienne comme dans la théogonie grecque la transgression n'est donc pas condamnée, mais exaltée. Il faut une succession de transgressions pour créer et organiser le monde. Mais à la fin l'ordre est là, stable, éternel. Marduk et Zeus sont établis à jamais. On châtie ceux qui voudraient le remettre en question.

Une relecture de la Genèse à la lueur de ces deux épopées me paraît devoir infléchir la vision négative qu'on a du péché originel. Iahvé, ayant créé le monde et installé l'homme dans le jardin d'Eden, lui permet de manger de tout arbre du jardin, sauf de l'arbre de la science du bien et du mal : « du jour où tu en mangerais tu mourrais ». La faute est commise : le serpent pousse la femme à cueillir le fruit de l'arbre et à en manger avec l'homme. Iahvé chasse les coupables du jardin d'Eden. L'humanité est condamnée au travail, à la souffrance et à la mort. La transgression est châtiée. Mais prenons-y garde : si le serpent a menti quand il a déclaré à la femme que manger le fruit ne provoquerait pas leur mort, il n'a pas menti quand il a ajouté : «le jour où vous en mangerez, vos yeux se dessilleront et vous serez comme des dieux, sachant le bien et le mal ». La preuve en est ce que dit Yahvé après le jugement de condamnation :

Voici que l'homme est devenu comme l'un de nous, grâce à la science du bien et du mal ! Maintenant il faut éviter qu'il étende sa main, prenne aussi de l'arbre de vie, en mange et vive à jamais. (Genèse, III, 22)

Certes, il y a eu châtiment, mais aussi instauration de l'humanité, qui n'est plus constituée de bêtes brutes, mais d'êtres conscients ayant part à la connaissance. La transgression du fruit cueilli est donc un acte fondateur. Il y a un prix à payer pour une acquisition essentielle. De la même façon, Hésiode présente deux transgressions fondamentales : celle de Zeus, détrônant son père pour instaurer le nouvel ordre cosmique; celle de Prométhée qui vole le feu pour le donner aux hommes. Hésiode présente cette seconde transgression comme 
entièrement négative et regrette le temps de l'âge d'or. Mais, reprenant le mythe, Eschyle exalte l'acte fondateur de Prométhée:

Écoutez en revanche les misères des mortels, et comment des enfants qu'ils étaient j'ai fait des êtres de raison, doués de pensée. (Esch., $P r$. 442-444)

Zeus fait payer cher au fils de Titan (Hésiode) ou Titan (Eschyle) sa philanthropia, mais l'humanité n'en est pas moins engagée sur la voie de la civilisation.

De cette première série on retiendra deux éléments : 1 . Des transgressions originelles ont organisé le monde ou donné à l'homme la conscience. 2. Pour une acquisition fondamentale il y a un prix à payer.

\section{La transgression cosmique : la remise en question de l'ordre}

Une fois que le dieu a instauré l'ordre cosmique, il n'est plus question qu'un transgresseur, qu'il s'appelle Lucifer, Samaël, Lilith ou Typhon, réintroduise le désordre originel. Il n'empêche que le roi des dieux doit toujours rester vigilant s'il veut préserver l'intégrité du cosmos : les puissances du Chaos ne restent pas toujours prisonnières du Schéol ou du Tartare. La mythologie grecque est riche de transgresseurs cosmiques. On peut distinguer trois catégories ${ }^{3}$ :

1. Les héros des théomachies. Des puissances monstrueuses tentent d'escalader le Ciel en entassant montagne sur montagne (l'Ossa sur l'Olympe et le Pélion sur l'Ossa), crachent le feu et lancent rocs et chênes enflammés vers les dieux. Elles sont en général foudroyées par Zeus et précipitées dans le Tartare: l'anabase s'inverse en katabase. La mythologie grecque compte quatre théomachies : la Titanomachie, la Gigantomachie, la Typhonomachie, le combat des Aloades, Otos et Éphialtès, des Géants, fils de Poséidon, qui osèrent déclarer la guerre aux dieux. De ces puissances monstrueuses on rapprochera cinq des sept chefs argiens de l'expédition contre Thèbes dans la version eschyléenne ${ }^{4}:$ le bestial Tydée, porteur d'un bouclier qui ressemble à un cosmos inversé; le braillard et menaçant Capanée; Étéoclos, dont le blason dit la volonté de s'élancer vers le ciel; Hippomédon, masse gigantesque plutôt qu'être humain; l'hybride Parthénopée, sauvage et cruel.

2. De jeunes héros présomptueux, qui présument de leurs forces, commettent des imprudences, s'abandonnent à la démesure : Bellérophon qui, avec Pégase, son cheval ailé, voulut atteindre la demeure de Zeus; Icare qui, pourvu

3 Voir Alain MOREAU, Ronsard: variations sur les mythes de l'anabase présomptueuse, in K. Christodoulou (éd.), Ronsard et la Grèce. Actes du Colloque d'Atbènes et Delphes, 4-7 Oct. 1985, Paris, 1988, p. 55-73

4 Voir A. MOREAU, Escbyle. La violence et le Chaos, Paris, Les Belles Lettres, 1985, p. 140-146. 
d'ailes fixées avec de la cire, s'approcha trop près du soleil; Phaéthon, imprudent conducteur du char de son père, Hélios, qui faillit provoquer la conflagration de l'Univers. Tous finissent foudroyés, renversés, estropiés, écrasés ou noyés.

3. Des personnages entre mythe et histoire, comme Cyrus qui, pour se venger du fleuve Gyndès qui avait englouti un des chevaux blancs du char du Soleil, renonce temporairement à la marche sur Babylone et utilise l'ensemble de son armée pendant plusieurs mois au creusement de trois cent soixante canaux qui divisent le cours du Gyndès ${ }^{5}$; naturellement, le châtiment d'une telle at teinte à l'ordre de la nature ne se fait pas attendre: Cyrus le conquérant lance peu après une expédition contre les Massagètes où il trouve une mort sanglante. Comme Xerxès chez Hérodote quand, parce qu'une violente tempête a disloqué les ponts jetés sur la mer punit celle-ci en ordonnant de frapper l'Hellespont de trois cents coups de fouet, de lancer dans son cours une paire d'entraves comme pour l'asservir, et même de le marquer au fer ${ }^{6}$. Et plus encore le Xerxès des Perses d'Eschyle, résurgence de Typhée/Typhon, monstrueux lieur aux mille bras et au « regard bleu sombre du dragon sanglant ${ }^{7}$. La défaite est la sanction de l'bubris.

\section{Une transgression primordiale : voir ce qu'il ne faut pas voir}

Il y a un regard de l'bubristès qui est une usurpation du regard divin ${ }^{8}$. Il n'est pas étonnant que chez tous les bubristai d'Eschyle on retrouve dans l'évocation du regard la racine indo-européenne derk, qui caractérise le regard fixe, perçant, funeste, du drakôn, serpent ou dragon, de Balor Birugderk qui pétrifie comme Méduse ${ }^{9}$, d'êtres monstrueux donc, mais aussi des dieux comme Zeus ou Athéna Oxuderkès : Xerxès, les chefs argiens, Clytemnestre, Prométhée et l'ambivalent Oreste. La faute de Prométhée est d'avoir ouvert les yeux des hommes :

Je leur rendis clairs ( $\dot{\xi} \xi \omega \mu \mu \alpha \dot{\alpha} \tau \omega \sigma \alpha)$ les signes de flamme jusque-là enveloppés d'ombre. (Esch., Pr., 498-499)

Voir, c'est déjà tomber dans la démesure. « Le regard, qui assure à notre conscience une issue hors du lieu qu'occupe notre corps, constitue, au sens le

\footnotetext{
5 HDT, I, 189-190.

6 HDT, VII, 33-35.

7 ESCH., Pe, 81-83. Voir MOREAU, Escbyle, op. cit. (n. 4), p. 147-148.

8 Voir A. MOREAU, L'ceil maléfique dans l'œuvre d'Escbyle, in REA, 78-79 (1976-1977), p. 50-64.

9 Voir MOREAU, L'eill maléfique, art. cit. (n. 8), p. 54-55. Balor est l'adversaire de Lugh dans la seconde bataille de Moytura (ou Mag-Tured), épopée irlandaise.
} 
plus rigoureux, un excès », écrit Jean Starobinski ${ }^{10}$. Tirésias a vu (involontairement, mais le crime n'en reste pas moins impardonnable) ce qu'il ne fallait pas voir : le corps dénudé d'Athéna. Dans une autre version, qui constitue une équivalence, il a appris ce qu'il ne fallait pas savoir : que pour dix parts de plaisir dans l'acte sexuel les femmes jouissent de neuf et l'homme d'une seule. Cette vision, ce savoir se paient de la perte de la vue ${ }^{11}$. Actéon a commis le même crime que Tirésias : il a vu le corps dénudé d'Artémis. La déesse le métamorphose en cerf et il est dévoré par ses propres chiens ${ }^{12}$. Dans une autre version, celle d'Acousilaos ${ }^{13}$, il avait provoqué la colère de Zeus parce qu'il voulait épouser Sémélé. Cette équivalence entre vision interdite et rivalité présomptueuse avec un dieu souligne bien le caractère de transgression du regard. Psyché, poussée par de mauvaises sœurs, commet la même faute en regardant, malgré l'interdiction, le corps du dieu Éros; elle le paie de cruelles épreuves ${ }^{14}$. Dans la légende poitevine de Mélusine, issue d'un vieux fonds indo-européen et plus particulièrement celtique ${ }^{15}$, le mythème est redoublé : le mortel Élinas a l'interdiction de voir la fée Pressine, son épouse, «en sa gésine », c'est-à-dire dans l'intervalle entre l'accouchement et les « relevailles ». Il enfreint l'interdit : il sera châtié avec la dernière rigueur. Lorsque ses filles sont âgées de quinze ans, Pressine leur révèle la faute du père et les filles, qui sont aussi des fées, indignées, jettent un sort à leur père; celui-ci finit sa vie enfermé dans une montagne. Dans un deuxième temps Mélusine, la fille aînée, épouse le comte Raimondin de Lusignan. Mais il a interdiction de la voir le samedi, car ce jour-là elle se métamorphose en serpente « de nombril en aval ». Poussé par un mauvais frère, le comte de Forez ${ }^{16}$, Raimondin enfreint l'interdiction et perd son épouse à jamais.

De même qu'il ne faut pas voir le corps de l'être surnaturel, il y a des corps d'êtres humains qu'il ne faut pas voir : ceux de parents proches. L'union

10 J. STAROBINSKI, L'Eil vivant, Paris, Gallimard, 1961, p. 14.

11 Sur toutes les versions et variantes, voir Luc BRISSON, Le mythe de Tirésias. Essai d'analyse structurale, Leiden, Brill, 1976.

12 PS. APD., Bibl., III, 4, 4.

13 Également rapportée par APOLLODORE = ACOUSILAOS, 2 F 33 Jacoby.

14 Voir ApulÉe, Mét., IV, 28 à VI, 24.

15 Sur Mélusine, voir Jean D'ARRAS, La Noble Histoire de Lusignan ou Le Roman de Mélusine en prose ou Le Livre de Mélusine en prose, vers 1400 (mis en français moderne par Michèle PERRET, avec préface de Jacques LE GOFF et postface de Michèle PERRET, Paris, Stock Plus, 1979). Version en vers, une dizaine d'années plus tard, par COULDRETTE sous le titre Le Roman de Lusignan et de Partbenay ou Mellusine. - Études: Georges PILLARD, Les thèmes initiatiques dans les romans de Mélusine, in Mélanges de Mytbologie française offerts à Henri Dontenville, Paris, Maisonneuve et Larose, 1980, p. 218-245; Laurence HARF-LANCNER, Une Mélusine galloise : la Dame de Brechnock, in Mélanges Jeanne Lods, Paris, 1978, p. 323-338; EAD., Les fées dans la littérature française au moyenâge. Morgane et Mélusine, Paris, Champion, 1991.

16 Le mythème des frères et des sceurs envieux et donneurs de mauvais conseils est fort répandu : on le trouve également dans le conte de La Belle et la Bête. 
incestueuse ou considérée comme telle est prohibée et entraîne le châtiment, qui peut prendre la forme de l'aveuglement ${ }^{17}$. C'est ce que subissent (Edipe, Aegypios ${ }^{18}$ (inceste avec la mère), Phonix (union réelle ou supposée avec la concubine du père, qui lui crève les yeux) ${ }^{19}$, les fils de Phinée (union supposée avec la belle-mère) ${ }^{20}$. Cette prohibition de la vue explique que tant de voyants soient aussi des aveugles : il faut payer très cher un don qui est normalement l'apanage des dieux. On voit ainsi avec amusement les mythographes déployer des trésors d'ingéniosité (qui les conduisent parfois à l'incohérence) pour expliquer l'aveuglement de Phinée alors que c'est son statut de devin qui constitue l'explication la plus vraisemblable ${ }^{21}$. Des héros qui n'ont pas le statut de professionnel de la divination deviennent des voyants à la suite de l'aveuglement : c'est en perdant la vision physique qu'CEdipe devient un voyant; naguère ennemi du devin aveugle Tirésias, il en devient le double. Polymestor, cupide et cruel roi de Thrace, à coup sûr fort éloigné de la sagesse et du sacré, une fois qu'il a été aveuglé par les servantes d'Hécube, se met à prophétiser comme une autre Cassandre ${ }^{22}$.

17 Voir Georges DevereuX, The Self-Blinding of Oidipous in Sophokles: Oidipous Tyrannos, in JHS, 93 (1973), p. 36-49.

18 ANTONinus libéralis, Métam., 5. Dans le cas d'Ægypios l'aveuglement est évité au dernier moment : lorsque Boulis, la mère, découvre qu'elle s'est unie à son propre fils, elle songe à lui arracher les yeux et à se donner la mort. Mais Zeus transforme tous les protagonistes de l'histoire en oiseaux.

19 Ps. APD., Bibl., III, 13, 8. L'édition Frazer donne les principales sources. Dans la version de l'lliade (IX, 437-484) Phœenix n'est pas aveuglé mais maudit.

20 Ps. APD., Bibl., I, 9, 21 (où l'édition Frazer donne les principales sources) et III, 15, 3.

21 Il a préféré une vie longue à la vue. Il avait le choix entre avoir le don de prophétie et être aveugle ou bien vivre peu de temps et ne pas avoir le don de prophétie mais garder son intégrité physique; il choisit la divination; Apollon l'aveugle. Il a révélé sa route à Phrixos (comprendre les fils de Phrixos): Hélios l'aveugle sur la demande de son fils Aiétès. Apollon lui a enseigné l'art divinatoire et par pitié pour les hommes il leur a révélé les desseins de Zeus; c'est pourquoi Zeus lui a envoyé un triple châtiment: la cécité, une vieillesse interminable, la persécution des Harpyes. Sur dénonciation calomnieuse de sa seconde épouse, il a aveuglé les enfants de la première épouse, Cléopâtre; c'est pourquoi il est aveuglé et persécuté par les Harpyes. Il a tué les enfants de Cléopâtre sur dénonciation de sa seconde épouse; Zeus en colère l'invite à choisir entre mourir ou devenir aveugle; il choisit de ne plus voir la lumière, c'est-à-dire Hélios; indigné, celui-ci lui envoie les Harpyes, etc. Voir A. MOREAu, La tétralogie des Perses a-t-elle une unité ?, in CGITA, 7 (1992-1993), p. 119-144 (126-127).

22 Voir EuR., Hécube, 1257-1284. Je connais bien des hellénistes qui, en examinant méthodiquement les passages de l'lliade où intervient Calchas, découvrent avec stupéfaction que jamais Homère ne fait allusion à sa cécité, tant la liaison entre cécité et divination est perçue comme fondamentale. 


\section{La transgression du territoire interdit. Fouler ce qui ne doit pas être foulé}

Il y a des actes (violence sexuelle, meurtre) qu'il est abominable de commettre dans un lieu consacré à une divinité. De tels actes entraînent irrévocablement le châtiment :

- Mélanion / Hippoménès s'unit à Atalante dans le sanctuaire de Zeus / Cybèle : tous les deux sont métamorphosés en lions (d'après les Anciens les lions ne s'unissaient pas entre eux : le lion s'unit au léopard femelle, la lionne au léopard mâle; le châtiment n'est donc pas seulement une animalisation, mais aussi une séparation) $)^{23}$.

- À Aulis, Agamemnon, qui s'est éloigné un peu du camp des Achéens, aperçoit une chèvre sauvage paissant dans un lieu consacré à Artémis. Sans se préoccuper de scrupules religieux, il la frappe de son épée. Artémis le lui fera payer du sacrifice de sa fille Iphigénie ${ }^{24}$.

- Ajax arrache Cassandre au Palladion, statue d'Athéna, dans le temple de la déesse à Troie, au moment du sac de la ville (version ancienne). Il viole la prophétesse au pied du Palladion (version récente). Il sera foudroyé par Athéna ou noyé par Poséidon ${ }^{25}$.

- Le Xerxès d'Eschyle et d'Hérodote est aussi un transgresseur. Lui, le souverain d'un empire terrestre, ose s'aventurer sur la mer, un élément qui lui est interdit. Lui, le souverain d'un empire asiatique, ose pénétrer en Europe. Puissance du Chaos, en jetant des ponts sur la mer, il a mêlé les éléments, transformé en terre ce qui était eau; inversement, en creusant le canal de l'Athos, il transforme en eau ce qui était la terre ${ }^{26}$.

\section{La parole sacrilège. Dire ce qu'il ne faut pas dire}

- Agamemnon, ayant atteint d'un trait un cerf, déclare : «Pas même Artémis » (ne pourrait faire aussi bien) ${ }^{27}$.

23 Voir Ps. APD., III, 9, 2 avec les principales sources dans l'éd. Frazer.

24 DICTYS DE CRËTE, I, 19.

25 Pour les sources, voir A. MOREAU, Les ambivalences de Cassandre, in Annie-France LAURENS (éd.), Entre bommes et dieux. Le convive, le béros, le propbète, Besançon, 1989 (Lire les polythéismes, 2), p. $147-167$.

26 La transgression se retrouve dans les contes, quand, par exemple, la jeune épouse de Barbe Bleue ouvre la porte du cabinet où sont pendus les corps des épouses précédentes : elle foule le territoire interdit, elle voit ce qu'elle ne doit pas voir. Dans le conte de Mme Leprince de Beaumont, la Belle et la Bête, le père de la Belle commet la transgression en cueillant une rose dans le jardin de la Bête.

27 Ps. APD., Épitomé, III, 21. Variante: « Même Artémis n'aurait pu décocher sa flèche de cette façon » (schol. Il., I, 108). 
- Ajax a eu «des pensers qui ne sont pas d'un homme ${ }^{28}$, et, faute plus grave encore, il les a exprimés. À son père qui l'invitait à son départ à souhaiter au combat la victoire, mais toujours la victoire avec l'aide d'un dieu, il a répondu, insolemment, follement :

Avec l'aide d'un dieu, père, cette victoire, même un homme de rien la pourrait obtenir. C'est sans les dieux que, pour ma part, je suis bien sûr de ramener la gloire. (Soph., $A j ., 767-769$ )

Puis, redoublant et aggravant sa faute, à Athéna qui l'invite à tourner son bras meurtrier du côté de l'ennemi, il fait une réponse « effrayante, inouïe »:

Va assister, maîtresse, les autres Argiens, ce n'est pas où je suis que le front craquera. (Soph., Aj., 774-775)

La parole interdite lui vaudra la folie, le déshonneur et la mort.

Bien entendu l'bubris des chefs argiens contre 'Thèbes passe d'abord par la parole. Une parole dite, écrite, représentée.

- La parole dite : Capanée saccagera la ville, même si la foudre de Zeus s'abat devant lui. Hippomédon pousse une clameur de guerre et, plein d'Arès, «appelle la bataille comme une Thyiade en délire ». Parthénopée «jure par la javeline qu'il tient à son poing et que sa foi révère plus qu'une divinité, plus que ses yeux mêmes, qu'il ravagera la cité cadméenne, en dépit de Zeus ».

- La parole écrite : sur le blason de Capanée on lit : «J'incendierai la ville ». Sur le blason d'Étéoclos il est inscrit « qu'Arès lui-même ne le jetterait pas à bas de ce rempart ».

- La parole représentée : sur le blason d'orgueil de Tydée on voit le ciel resplendissant d'étoiles avec la lune en son plein. Sur celui de Capanée est représenté un porteur de feu, un purphoros, qui annonce l'incendie de la ville mais rappelle aussi Prométhée, le Titan ou fils de Titan adversaire de Zeus. Sur le blason d'Étéoclos un guerrier gravit les degrés d'une échelle appliquée au mur ennemi. Le blason d'Hippomédon procède de la même bubris puisqu'on y voit Typhée.

La parole impie peut être triplée à la manière des trois coups sacrilèges dont Clytemnestre frappe Agamemnon, son époux et son roi :

Et je frappe - deux fois - et, sans un geste, en deux gémissements, il laisse aller ses membres; et, quand il est à bas, je lui donne encore un troisième coup, offrande votive au Zeus Sauveur des morts qui règne sous la terre. (Esch., $\mathrm{Ag}$, 1384-1387) 
Trois héros sophocléens se rendent coupables de cette triplication du sacrilège. Aux trois personnages raisonnables qui tentent de l'empêcher de commettre l'acte d'bubris qui le conduira à la ruine, Créon répond par un défi à Zeus.

- au Coryphée : «Qu'elle soit née de ma sœur, qu'elle soit encore plus proche de moi / que tous ceux qui peuvent ici se réclamer du Zeus de notre maison, il n'importe : / ni elle ni sa sœur n'échapperont / à une mort infâme ». (Ant., 486-489)

- à Hémon : «Qu'elle invoque à son gré Zeus protecteur des droits du sang ». (Ant., 658-659)

- à Tirésias, à propos du cadavre de Polynice : «Non, quand les aigles de Zeus désireraient pour le manger / l'emporter jusques au trône du dieu, / même alors, ne comptez pas que, par crainte d'une souillure, / je vous laisse l'enterrer, moi ». (Ant., 1040-1042)

Les défis sacrilèges s'achèvent par une imprécation que le transgresseur lance contre lui-même, une fois que la catastrophe s'est abattue sur les siens :

"I $\tau \omega$, "̈ $\tau \omega$,

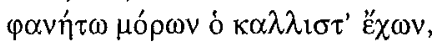

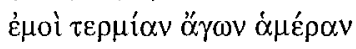
ט้ $\pi \alpha \tau \sigma \varsigma^{\prime}$ '

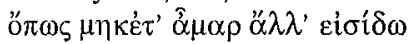

Qu'elle s'approche, qu'elle s'approche, Qu'elle se montre, la plus magnifique des morts, amenant pour moi le jour terminal, accomplissement; qu'elle s'approche, qu'elle s'approche, pour que jamais plus je ne contemple un seul jour.

$$
\text { (Soph., Ant., 1328-32) }
$$

Les répétitions et les allitérations ( $t \hat{o}, a, o, \hat{o}, m, r$; té, to, ta) rythment l'imprécation, soulignent la violence de la souffrance.

C'est vers le dieu Apollon que Jocaste lance ses défis.

- Persuadée que Laïos n'est pas mort sous la main de son fils comme l'avait prédit Apollon, elle s'écrie : «... désormais, en matière de prophéties, / je ne tiendrai pas plus compte de ceci que de cela ». (O.R., 857-858)

- Apprenant la mort de Polybe : «Ah ! oracles divins, où êtes-vous donc à cette heure?» (946-947). Et quelques vers plus loin :

- «Écoute l'homme qui est là, et vois en l'écoutant ce que sont devenus ces oracles augustes d'un dieu ». (952-953)

Comme pour Créon les défis aux dieux s'achèvent par le désastre et une douloureuse imprécation de Jocaste contre elle-même. Elle lance un goos qu'il 
faut comprendre, plutôt que comme un chant plaintif, comme une véritable incantation de sorcier (le goètès), scandée par les répétitions et les allitérations :

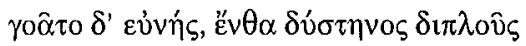

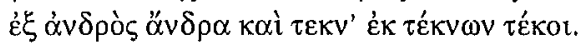

Elle lance une lugubre incantation contre sa couche, « où, double détresse, de son époux un époux, de ses enfants des enfants elle enfanta!»

$$
\text { (Soph., O.R., 1249-50) }
$$

Cdipe injurie le prêtre d'Apollon, défie Apollon lui-même, mais aussi l'ensemble des dieux, appelant sur lui tous les malheurs du monde :

- «... ce faux prophète, ce grand meneur d'intrigues, ce fourbe charlatan... » (387-388)

- «Ah! femme, qui pourrait désormais recourir / à Pythô, au foyer prophétique? ou bien / à ces oiseaux criaillant sur nos têtes ?» (964-966)

- «Eh! qu'éclatent donc tous les malheurs qui voudront! ... je me tiens, moi, pour fils de la Fortune, Fortune la généreuse, et n'en éprouve point de honte. » (1076 sq.)

Les défis sacrilèges aux dieux débouchent sur la condamnation du héros par lui-même :

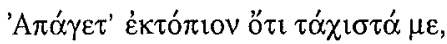

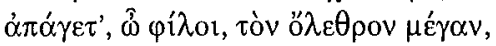

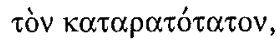

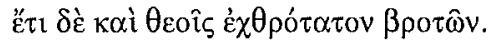

Conduisez-moi hors de ce territoire, très vite,

conduisez, mes amis, cette funeste ruine,

cet être totalement exécré,

maintenant enfin pour les dieux le plus détesté des mortels!

(O.R., 1340-45)

Là encore on dirait une formule d'exécration, une formule magique incompréhensible, avec ses répétitions de mots et de syllabes, ses consonnes agressives, gutturales, dentales et rhô, ses alpha retentissants, aux sons d'autant plus rudes et éclatants qu'il s'agit d'agir sur les puissances du Mal enfouies au plus profond des Enfers pour les mettre au service de l'Imprécateur. On pourrait aisément rapprocher les imprécations des héros sophocléens de formules prises au hasard dans l'ouvrage d'André Bernand ${ }^{29}$ :

29 André BERNAND, Sorciers grecs, Paris, Fayard, 1991, p. 342 : "Dans la liste des "mots barbares", la recherche de l'allitération et de l'homoïotéleute est évidente». 


\section{AZARACHTHARAEZA LATHA IATHAL YYYY LATHAI \\ LAKI LAKIOLAKIMOU MOUKILA KILAMOU \\ ERÊKISITHPHRÊ ARARARACHARARA EPHTHISIKÊRE \\ CHAÔR, CHTHÔR, CHARARBA, CHOLBAS, CHTHRYTHYR}

Comme les sorciers grecs ou égyptiens entrant en contact avec les puissances infernales, les souverains thébains frappés par le châtiment divin sont en relation avec la mort : Créon parce qu'il revient de la caverne souterraine où Antigone s'est pendue et où son fils Hémon s'est frappé au flanc de son épée; Jocaste, parce qu'elle va se pendre; CEdipe, parce qu'il s'apprête à entrer dans la nuit.

\section{Le culte sacrilège. Vénérer ce qu'il ne faut pas vénérer}

Une forme particulière de la transgression est la profanation du sacré qui consiste à remplacer le culte rendu aux dieux par un culte rendu aux armes. Un tel comportement est bon pour des Barbares, Scythes et Sauromates adorant leur cimeterre (akinakès), peuplades indigènes commandées par Mézence ou Turnus adorant leur lance. Mais même dans ce cas les adorateurs ne peuvent être que des bubristai : Mézence est comparé à Orion, Turnus est agité par les Furies, son visage jette des étincelles, le feu brille dans ses yeux. Ils sont, bien sûr, promis à la mort, comme les Grecs qui commettent cette profanation et se rabaissent au niveau des non-Grecs : Caineus, roi des Lapithes, qui contraint son peuple à vénérer son javelot fiché au centre de la place publique, Parthénopée et Idas, qui vénèrent leur lance; comme aussi un personnage historique, Alexandre, tyran de Phères en Thessalie qui «avait consacré et couronné de fleurs la lance avec laquelle il avait tué son oncle Polyphron et lui sacrifiait comme à une divinité, qu'il appelait Tychon ». Perfide, lâche et féroce, il finit sous les coups de ses trois beaux-frères guidés par sa propre épouse. Son cadavre est jeté dehors, foulé aux pieds et mutilé par ses sujets ${ }^{30}$.

30 Scythes et Sauromates : CLÉMENT D'ALEXANDRIE (citant EUdOXE), Protr., V, 56P; LUCIEN, Toxaris ou l'amitié $(=$ Tox.), 38; ID., Zeus Tragique $(=J . t r)$, 42; Mézence et Turnus : VIRG., Én., X, 773774, XII, 95-100; leur bubris : Én., X, 763 sq., XII, 101 sq.; Caineus : ACOUSILAOS, 2 F 22 Jacoby; Parthénopée: ESCH., Se., 529-532 (voir supra); Idas: AP. RH., I, 466-468. Sur Idas voir maintenant la toute récente étude de Jean-Michel RENAUD, L'v̋ $\beta \rho ı \zeta$ dans le mytbe d'Idas et de Lyncée, in Uranie, 6 (1996), p. 49-60. On notera que Caineus et Idas sont tous les deux fils de Poséidon, dieu à la fois chthonien et olympien, qui déchaîne les tremblements de terre et les tempêtes, se trouve souvent en conflit avec Zeus et se caractérise par une descendance d'bubristai : Caineus, Idas, le Géant Orion, le monstrueux Polyphème, les Aloades, Otos et Éphialtès, héros d'une théomachie (enfermement d'Arès dans une jarre de bronze, tentative de viol sur Artémis, tentative d'escalade de l'Olympe grâce à l'entassement du Pélion sur l'Ossa. Voir plus haut sur les théomachies, et RENAUD, art. cit., p. 53-55). Alexandre de Phères: PluT., Pél, 26-35. 


\section{Conclusion}

Ce panorama des transgressions majeures permettra peut-être de préciser quelque peu la notion d' bubris. Il est clair que la transgression, franchissement de la frontière interdite, est essentielle. "Passer outre » entraîne nécessairement une sanction. Mais il me semble qu'en filigrane une autre notion apparentée se fait jour, la monstruosité. Transgresser, c'est ne pas respecter la norme, être hors norme, autrement dit anormal. L'anormalité morale est souvent rendue visible par l'anormalité physique. Les bubristai sont des monstres moraux et fort souvent des monstres physiques par leur gigantisme, leurs membres proliférants, leur hybridité. On ne s'étonnera donc pas de retrouver derrière la violence sauvage des chefs argiens devant Thèbes celle des Géants de la Gigantomachie, derrière la démesure de Xerxès l'ombre de Typhon le proliférant. Chantraine déclare que l'étymologie d'ü $\beta \rho$ ş est inconnue. Mais il y a de fausses étymologies qui sont fort éclairantes. Des hellénistes ont rapproché le mot d'v́rép, ce qui est

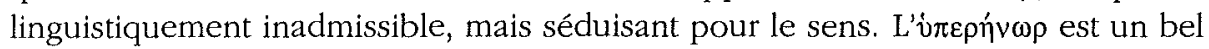

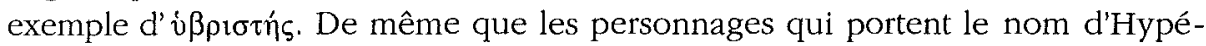
rénor : l'un est un Troyen qui injurie Ménélas (et trouve très vite la mort), l'autre l'un des cinq Spartes nés des dents du dragon tué par Cadmos et ayant échappé au massacre réciproque : c'est un géant monstrueux ${ }^{31}$. Une seconde étymologie également fausse, mais tout aussi parlante, est celle du mot bybride, dont la première occurrence date de 1596. Dauzat explique que ce terme vient du latin bibrida, «de sang mêlé », altéré en bybrida sous l'influence du grec bubris, «violence ». La violence se trouve ici associée au mélange, au désordre, au monstrueux, car l'une des caractéristiques les plus fréquentes de la monstruosité, c'est précisément l'hybridité : il suffit d'examiner la descendance de Méduse dans la Théogonie ${ }^{32}$. Les fausses étymologies sont souvent très éclairantes, comme celle qui métamorphose le forsené (foris sensu), hors de son sens, de la Vie de Saint Alexis en forcené, que sa force empêche de maîtriser, à la Renaissance.

Le champ sémantique de l'bubris est donc large puisqu'il comporte la transgression, la violation, le sacrilège, la violence, la démesure, l'excès, le gigantisme, le désordre, le mélange, l'hybridité, le monstrueux. Tous ces termes sont de tonalité négative. Et pourtant, nous l'avons vu, la transgression peut être féconde. Que serait l'ordre du monde sans les révoltes de Marduk et de Zeus contre les souverains anciens? Que serait l'homme de la Bible sans l'intervention du serpent? Un être sans savoir, une ombre aussi ombre que les eidôla du Chant XI de l'Odyssée. Que serait l'homme de la Grèce sans Prométhée? Le

31 Le Troyen : $1 l$, XIV, 516-519, XVII, 24-28; le Sparte : 1re attestation, HeLlaNICOS, 4 F 1a Jacoby. Voir Paul WATHELET, Dictionnaire des Troyens de l'lliade, Univ. de Liège, 1988, II, p. 1035-1036.

32 Voir A. MOREAU, La race de Méduse: forces de vie contre forces de mont (Hésiode, Théogonie, $v, 270-336$ ), in François JOUAN (éd.), Mort et fécondité dans les mythologies. Actes du Colloque de Poitiers, 13-14 Mai 1983, Paris, Les Belles Lettres, 1986, p. 1-17. 
philantbrope a apporté aux hommes la connaissance (comme le prouvent les vers 442-444 du Prométbée cités plus haut) et l'organisation :

Au début ils voyaient sans voir, ils écoutaient sans entendre, et, pareils aux formes des songes, ils vivaient leur longue existence dans le désordre et la confusion. (Esch., Pr, 446-450)

Il est clair ici que, paradoxalement, la transgression, le refus de l'ordre ancien, sont au service de l'ordre. Mais, après tout, le désordre est-il toujours condamnable? Pour l'auteur de la République, la démocratie athénienne est un $\pi \alpha v \tau o \pi \omega \dot{\lambda}$ เov, un bazar, un souk. On peut néanmoins préférer la liberté désordonnée d'Athènes à l'ordre orwellien de la cité platonicienne.

Alain MOREAU

Université Paul Valéry, Montpellier

Route de Mende - BP 5043

F- 34032 MONTPELLIER Cedex 1 